\title{
Correction to: A Pattern Recognition Tool for Medium-Resolution Cryo-EM Density Maps and Low-Resolution Cryo-ET Density Maps
}

Devin Haslam, Salim Sazzed, Willy Wriggers, Julio Kovacs, Junha Song, Manfred Auer, and Jing He

\section{Correction to:}

Chapter "A Pattern Recognition Tool for Medium-Resolution Cryo-EM Density Maps and Low-Resolution Cryo-ET Density Maps" in: F. Zhang et al. (Eds.): Bioinformatics

Research and Applications, LNBI 10847, https://doi.org/10.1007/978-3-319-94968-0_22

The original version of this chapter contained an error in the fourth author's name. The spelling of Julio Kovacs's name was incorrect in the header of the paper. The author name was corrected. 